\title{
Radiatively Generated Maximal Mixing Scenario for the Higgs Mass and the Least Fine Tuned Minimal Supersymmetric Standard Model
}

\author{
Radovan Dermíšek ${ }^{1}$ and Hyung Do Kim ${ }^{2}$ \\ ${ }^{1}$ School of Natural Sciences, Institute for Advanced Study, Princeton, NJ 08540, U.S.A. \\ ${ }^{2}$ School of Physics and Center for Theoretical Physics, \\ Seoul National University, Seoul, 151-747, Korea
}

(Dated: January 5, 2006)

\begin{abstract}
We argue that given the experimental constraints on the Higgs mass the least fine tuned parameter space of minimal supersymmetric standard model is with negative stop masses squared at the grand unification scale. While stop mass squared is typically driven to positive values at the weak scale, the contribution to the Higgs mass squared parameter from the running can be arbitrarily small, which reduces fine tuning of electroweak symmetry breaking. At the same time the stop mixing is necessarily enhanced and the maximal mixing scenario for the Higgs mass can be generated radiatively even when starting with negligible mixing at the unification scale. This highly alleviates constraints on possible models for supersymmetry breaking in which fine tuning is absent.
\end{abstract}

Minimal Supersymmetric Standard Model (MSSM) is a promising candidate for describing physics above the electroweak (EW) scale. The three gauge couplings unify at the GUT (grand unified theory) scale $\sim 2 \times 10^{16} \mathrm{GeV}$ within a few percent, and the hierarchy between the EW scale and the GUT scale is naturally stabilized by supersymmetry (SUSY). In addition, if we add softsupersymmetry-breaking terms at the GUT scale we typically find that the mass squared of the Higgs doublet which couples to the top quark $\left(H_{u}\right)$, is driven to negative values at the EW scale. This triggers electroweak symmetry breaking and the EW scale is naturally understood from SUSY breaking scale. Furthermore, assuming R-parity, the lightest supersymmetric particle (LSP) is stable and it is a natural candidate for dark matter of the universe.

The real virtue of supersymmetry is that the above mentioned features do not require any specific relations between soft-supersymmetry-breaking parameters (SSBs) and the only strong requirement on SUSY breaking scenarios is that these terms are of order the EW scale. However generic SSBs near the EW scale generically predict too light Higgs mass which is ruled out by LEP limits. The exact value of the Higgs mass is not relevant for low energy physics, nothing crucially depends on it, and yet, in order to stay above LEP limits $\left(m_{h} \gtrsim 114.4\right.$ $\mathrm{GeV}$ [1]) the SSBs have to be either considerably above the EW scale or related to each other in a non-trivial way. SSBs can no longer be just generic which leads to strong requirements on possible models for SUSY breaking should these provide a natural explanation for the scale where electroweak symmetry is broken.

In this letter we show that such constraints are highly alleviated and the fine tuning is in principle absent in scenarios which have negative stop masses squared at the unification scale. While stop mass squared is typically driven to positive values at the EW scale by gluino loops through renormalization group (RG) running, the contribution to the Higgs mass squared parameter from the running (mostly due to top Yukawa coupling) can be arbitrarily small, which reduces fine tuning of electroweak symmetry breaking. At the same time the stop mixing is necessarily enhanced which is known to enlarge the Higgs mass. Even the maximal mixing scenario for the Higgs mass can be radiatively generated (starting with negligible mixing at the GUT scale). Thus in the least fine tuned scenarios the Higgs mass is highly enhanced without any further assumptions.

In spite of having tachyonic scalar masses at a high scale such scenarios are not excluded by our current knowledge of cosmology. We discuss constraints from charge and color breaking minima on possible scenarios. Finally, we discuss a typical spectrum of these scenarios which is characterized by light stop, light higgsino and a fairly light gluino.

The tension between the direct search bound on the Higgs mass and naturalness of electroweak symmetry breaking can be summarized as follows 2]. At tree level, the mass of the lightest Higgs boson in MSSM is bounded from above by the mass of the $\mathrm{Z}$ boson,

$$
m_{h}^{2}<M_{Z}^{2} \cos ^{2} 2 \beta,
$$

where $\tan \beta=v_{u} / v_{d}$ is the ratio of the vacuum expectation values of $H_{u}$ and $H_{d}$. The dominant one loop correction, in case the stop mixing parameter is small, is proportional to $m_{t}^{4} \log \left(m_{\tilde{t}}^{2} / m_{t}^{2}\right)$ (for simplicity we assume $m_{\tilde{t}} \simeq m_{\tilde{t}_{L}} \simeq m_{\tilde{t}_{R}}$ throughout this paper). It depends only logarithmically on stop masses and it has to be large in order to push the Higgs mass above the LEP limit. A two loop calculation (we use FeynHiggs 2.2.10 [3, 4] with $m_{t}=172.7 \mathrm{GeV}$ ) reveals the stop masses have to be $\gtrsim 900 \mathrm{GeV}$. On the other hand, the mass of the $\mathrm{Z}$ boson $\left(M_{Z} \simeq 91 \mathrm{GeV}\right)$ is given from the minimization of the scalar potential as (for $\tan \beta \gtrsim 5$ )

$$
\frac{M_{Z}^{2}}{2} \simeq-\mu^{2}\left(M_{Z}\right)-m_{H_{u}}^{2}\left(M_{Z}\right)
$$


and the large stop masses directly affect the running of soft scalar mass squared for $H_{u}$,

$$
\delta m_{H_{u}}^{2} \simeq-\frac{3}{4 \pi^{2}} m_{\tilde{t}}^{2} \log \frac{\Lambda}{m_{\tilde{t}}} .
$$

Numerically the loop factor times large log is of order one for $\Lambda \sim M_{\mathrm{GUT}}$ and we have $\delta m_{H_{u}}^{2} \simeq-m_{\tilde{t}}^{2}$. Starting with negligible $m_{H_{u}}^{2}$ at the GUT scale we find $m_{H_{u}}^{2}\left(M_{Z}\right) \simeq$ $\delta m_{H_{u}}^{2} \simeq-m_{\tilde{t}}^{2} \simeq-(900 \mathrm{GeV})^{2}$ and the correct $\mathrm{Z}$ mass requires that $\mu^{2}\left(M_{Z}\right)$ is tuned to $m_{H_{u}}^{2}\left(M_{Z}\right)$ with better than one percent accuracy. Alternatively, we can start from large positive $m_{H_{u}}^{2}\left(M_{\mathrm{GUT}}\right) \sim(900 \mathrm{GeV})^{2}$ in which case $m_{H_{u}}^{2}\left(M_{Z}\right) \sim-M_{Z}^{2}$ is possible. However, in this case the fine tuning is hidden in $m_{H_{u}}^{2}\left(M_{\mathrm{GUT}}\right)$. Small change of the boundary condition $m_{H_{u}}^{2}\left(M_{\mathrm{GUT}}\right)$ would generate very different value for the EW scale and the situation is quite similar to the tuning of $\mu$.

The situation highly improves when considering large mixing in the stop sector. The mixing is controlled by the ratio of $A_{t}-\mu \cot \beta$ and $m_{\tilde{t}}$. Since we consider parameter space where $\mu$ is small to avoid fine tuning and $\tan \beta \gtrsim 5$ in order to maximize the tree level Higgs mass (1), the mixing is simply given by $A_{t} / m_{\tilde{t}}$. It was realized that mixing $A_{t}\left(M_{Z}\right) / m_{\tilde{f}}\left(M_{Z}\right) \simeq \pm 2$ maximizes the Higgs mass for given $m_{\tilde{t}}[\underline{5}$, while still satisfying constraints to avoid charge and color breaking (CCB) minima [6]. Using FeynHiggs 2.2.10 we find that $m_{\tilde{t}}\left(M_{Z}\right) \simeq 300 \mathrm{GeV}$ and $\left|A_{t}\left(M_{Z}\right)\right|=450 \mathrm{GeV}$ (for $\tan \beta \gtrsim 50$ ), $\left|A_{t}\left(M_{Z}\right)\right|=500$ (for any $\tan \beta \gtrsim 8$ ) or $\left|A_{t}\left(M_{Z}\right)\right|=600 \mathrm{GeV}$ (for $\tan \beta$ as small as 6) satisfies the LEP limit on the Higgs mass [22]. Therefore large stop mixing, $\left|A_{t}\left(M_{Z}\right) / m_{\tilde{t}}\left(M_{Z}\right)\right| \gtrsim 1.5$ is crucial for satisfying the LEP limit with light stop masses (the physical stop mass in this case can be as small as current experimental bound, $\left.m_{\tilde{t}_{1}} \gtrsim 100 \mathrm{GeV}\right)$. Decreasing the mixing requires increasing of $m_{\tilde{t}}$ and finally we end up with $m_{\tilde{t}} \gtrsim 900 \mathrm{GeV}$ for small mixing.

In order to discuss fine tuning in this case, the approximate solution of RG equation for $m_{H_{u}}^{2}$, Eq. (3), is not sufficient. For given $\tan \beta$ we can solve $\mathrm{RG}$ equations exactly and express EW values of $m_{H_{u}}^{2}, \mu^{2}$, and consequently $M_{Z}^{2}$ given by Eq. (2), as functions of all GUT scale parameters [7, [8]. For $\tan \beta=10$, we have:

$$
\begin{aligned}
M_{Z}^{2} \simeq & -1.9 \mu^{2}+5.9 M_{3}^{2}-1.2 m_{H_{u}}^{2}+1.5 m_{\tilde{t}}^{2} \\
& -0.8 A_{t} M_{3}+0.2 A_{t}^{2},
\end{aligned}
$$

where parameters appearing on the right-hand side are the GUT scale parameters, we do not write the scale explicitely. The contribution of $M_{2}$ to the above formula is small and when $M_{2} \sim M_{3}$ it cancels between $\simeq-0.4 M_{2}^{2}$ term and the mixed $\simeq 0.4 M_{3} M_{2}$ term. Other scalar masses and $M_{1}$ appear with negligible coefficients and we neglect them in our discussion. The coefficients in this expression depend only on $\tan \beta$ (they do not change dramatically when varying $\tan \beta$ between 5 and 50) and $\log \left(M_{G U T} / M_{Z}\right)$.
Let us also express the EW scale values of stop mass squared, gluino mass and top trilinear coupling for $\tan \beta=10$ in a similar way:

$$
\begin{aligned}
m_{\tilde{t}}^{2}\left(M_{Z}\right) & \simeq 5.0 M_{3}^{2}+0.6 m_{\tilde{t}}^{2}+0.2 A_{t} M_{3} \\
M_{3}\left(M_{Z}\right) & \simeq 3 M_{3} \\
A_{t}\left(M_{Z}\right) & \simeq-2.3 M_{3}+0.2 A_{t} .
\end{aligned}
$$

In the case of $m_{\tilde{t}}$ the coefficients represent averages of exact coefficients that would appear in separate expressions for $m_{\tilde{t}_{L}}^{2}$ and $m_{\tilde{t}_{R}}^{2}$.

In the limit when the stop mass, $m_{\tilde{t}}\left(M_{Z}\right) \simeq 300 \mathrm{GeV}$, originates mainly from $M_{3}$, from Eq. (5) we see we need $M_{3} \simeq 130 \mathrm{GeV}$. Then Eq. (7) shows that the necessary $\left|A_{t}\left(M_{Z}\right)\right| \simeq 500 \mathrm{GeV}$ is obtained only when $A_{t} \lesssim-1000$ $\mathrm{GeV}$ or $A_{t} \gtrsim 4000 \mathrm{GeV}$ at the GUT scale, in both cases it has to be signifficantly larger than other SSBs. The contribution from the terms in Eq. (4) containing $M_{3}$ and $A_{t}$ is at least $(600 \mathrm{GeV})^{2}$ and therefore large radiative correction has to be cancelled either by $\mu^{2}$ or $m_{H_{u}}^{2}\left(M_{G U T}\right)$. If $m_{\tilde{t}}$ is not negligible at the GUT scale, $M_{3}$ can be smaller, but in this case we need even larger $A_{t}$ and the conclusion is basically the same. The situation improved by considering large $A_{t}$ term. However, we still need at least $3 \%$ fine tuning.

Although $M_{Z}$ results from cancellations between SSBs [23] it does not mean that it is necessarily fine tuned. SUSY breaking scenarios typically produce SSBs which are related to each other in a specific way in which case we should not treat each one of them separately. Although, in this case, our conclusions about the level of fine tuning are irrelevant, the discussion above tells us what relations between SSBs have to be generated, should the $M_{Z}$ emerge in a natural way. For instance, it was recently discussed that fine tuning can be reduced with a proper mixture of anomaly and modulus mediation [9, 10, 11] which produces boundary conditions leading to large stop mixing at the EW scale and an initial value of $m_{H_{u}}^{2}$ canceling most of the contribution from running.

Even if SUSY breaking scenario produces SSBs related to each other in a way that guarantees large degree of cancellation, still they cannot be arbitrarily heavy because in that case the $M_{Z}$ much smaller than superpartner masses would emerge as a coincidence and we would not have a natural explanation for it. This "coincidence" problem is further amplified by the fact that the relations that have to be satisfied between SSBs in order to recover the correct $M_{Z}$ depend on the energy interval they are going to be evolved. Therefore a SUSY breaking scenario would have to know that SSBs will evolve according to MSSM RG equations, and exactly from $M_{G U T}$ to $M_{Z}$.

There is one possibility which to large extend overcomes this problem. If we allow negative stop masses squared at the GUT scale several interesting things happen simultaneously. First of all, from Eq. (5D) we see 


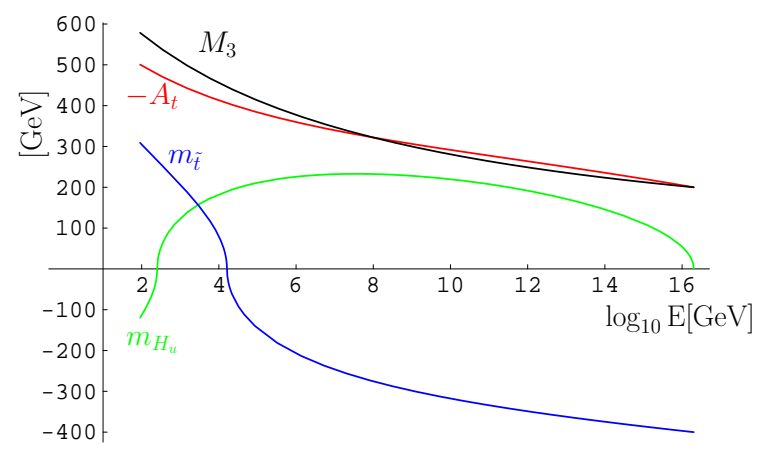

FIG. 1: Renormalization group running of relevant SSBs for $\tan \beta=10$ and GUT scale boundary conditions: $-A_{t}=M_{3}=$ $200 \mathrm{GeV}, m_{\tilde{t}}^{2}=-(400 \mathrm{GeV})^{2}$ and $m_{H_{u}}^{2}=0 \mathrm{GeV}^{2}$. In order to have both mass dimension one and two parameters on the same plot and keep information about signs, we define $m_{H_{u}} \equiv$ $m_{H_{u}}^{2} / \sqrt{\left|m_{H_{u}}^{2}\right|}$ and $m_{\tilde{t}} \equiv m_{\tilde{t}}^{2} / \sqrt{\left|m_{\tilde{t}}^{2}\right|}$.

that unless $m_{\tilde{t}}$ is too large compared to $M_{3}$ it will run to positive values at the $\mathrm{EW}$ scale. At the same time the contribution to $m_{H_{u}}^{2}$ from the energy interval where $m_{\tilde{t}}^{2}<0$ partially or even exactly cancels the contribution from the energy interval where $m_{\tilde{t}}^{2}>0$ and so the EW scale value of $m_{H_{u}}^{2}$ can be arbitrarily close to the starting value at $M_{G U T}$, see Fig. 1 From Eq. (4) we see that this happens for $m_{\tilde{t}}^{2} \simeq-4 M_{3}^{2}$ (neglecting $A_{t}$ ). No cancellation between initial value of $m_{H_{u}}^{2}$ (or $\mu$ ) and the contribution from the running is required. And finally, from Eqs. (5) and (7) we see that the stop mixing is typically much larger than in the case with positive stop masses squared. For positive (negative) stop masses

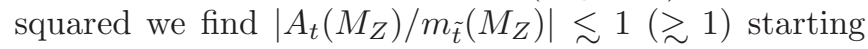
with $A_{t}=0$ and small $m_{\tilde{t}}$ at the GUT scale. Starting with larger $m_{\tilde{t}}$ the mixing is even smaller (larger) in the positive (negative) case. Therefore large stop mixing at the EW scale is generic in this scenario and actually it would require very large GUT scale values of $A_{t}$ to end up with small mixing at the EW scale.

It turns out that in the region where $m_{H_{u}}^{2}$ gets negligible contribution from running, the radiatively generated stop mixing is close to maximal even when starting with negligible mixing at the GUT scale. In this case, comparing Eqs. (5) and (7), we find 24]

$$
A_{t}\left(M_{Z}\right) / m_{\tilde{t}}\left(M_{Z}\right) \simeq-1.5+0.2 A_{t} / M_{3}
$$

Slightly more negative stop masses squared at the GUT scale would result in maximal stop mixing at the EW scale even when starting with negligible $A_{t}$. Nevertheless the example in Fig. 1 with simple GUT scale boundary conditions already leads to EW scale parameters $m_{\tilde{t}}\left(M_{Z}\right) \simeq 300 \mathrm{GeV}$ and $A_{t}\left(M_{Z}\right)=-500 \mathrm{GeV}$ producing sufficiently heavy Higgs boson, $m_{h} \simeq 115.4 \mathrm{GeV}$. Small variations of GUT scale parameters, including positive or negative values of $m_{H_{u}}^{2}$, would produce similar re- sults and scaling all parameters up would lead to larger Higgs mass.

In a theory which predicts $m_{\tilde{t}}^{2} \simeq-4 M_{3}^{2}$, the fine tuning problem is entirely solved. The contribution to $m_{H_{u}}^{2}$ from the running is negligible and the $\mathcal{O}\left(M_{Z}^{2}\right)$ values of $m_{H_{u}}^{2}$ and $\mu^{2}$ at the GUT scale naturally result in the correct $M_{Z}$. However, the absence of fine tuning is quite robust and the relation above does not have to be satisfied very precisely. If we define $\alpha$ by

$$
\frac{\left|m_{\tilde{t}}\right|}{M_{3}}=2(1+\alpha),
$$

then the EW scale (4) can be written as

$$
M_{Z}^{2} \simeq-1.9 \mu^{2}-1.2 m_{H_{u}}^{2}-12 \alpha M_{3}^{2}
$$

We see that requiring fine tuning less than 10\%, large range of $\alpha$ is allowed (for $M_{3} \simeq 200 \mathrm{GeV}$ ):

$$
-0.17<\alpha<0.17 \text {. }
$$

This interval is shrinking with increasing $M_{3}$ which is a sign of the coincidence problem discussed above.

In summary, a very reasonable set of SSBs at the GUT scale: $M_{3} \gtrsim 200 \mathrm{GeV},\left|m_{\tilde{t}_{L}}\right| \simeq\left|m_{\tilde{t}_{R}}\right| \simeq(1.7-2.3) M_{3}$ and $A_{t}$ of order the other SSBs or smaller naturally reproduces the correct EW scale. The EW scale value of $m_{H_{u}}^{2}$ is very close to the starting value at the GUT scale. In a simplified way this can be understood as effectively lowering the scale where SSBs are generated to the scale where $m_{\tilde{t}} \simeq 0$ (in the example in Fig. 1 it is $10 \mathrm{TeV}$ ). From this scale SSBs run in a similar way they would run when starting with positive stop masses. However this scale is much closer to the EW scale and so $\delta m_{H_{u}}^{2}$, Eq. (3), generated between this scale and the EW scale is considerably smaller. The stop mixing at the EW scale is close to maximal, but it is generated radiatively starting from a small mixing at the GUT scale. It is to be compared with the positive case which requires $A_{t}$ to be several times larger than other SSBs in order to produce large enough mixing to satisfy LEP bounds on the Higgs mass. Thus considering negative values for stop masses squared keeps the desirable feature of radiative electroweak symmetry breaking and minimizes fine tuning. The Higgs mass is automatically enhanced and staying above the LEP bound does not require additional constraints on the rest of SUSY parameters.

However strong constraints can originate when considering possible CCB minima. At the EW scale all scalar masses squared (except $m_{H_{u}}^{2}$ ) are positive, nevertheless, as already discussed, very large $A_{t}$ term would generate a CCB minimum at around the EW scale 12, 13. Then the EW vacuum should be the global minimum since otherwise the EW vacuum would rapidly tunnel to the CCB minimum as the barrier is neither high nor thick. The optimal sufficient condition to avoid a CCB vacuum in 
$\left(H_{u}, \tilde{t}_{L}, \tilde{t}_{R}\right)$ plane is $\left|A_{t}\right| \lesssim 2 m_{\tilde{t}}[\underline{6}]$. The generated $A_{t}$ in the region we consider (8) may be close but is typically well within this bound.

Negative stop masses squared at the GUT scale result in unbounded from below (UFB) potential along the Dflat direction [14, 15]. The tree level potential at the GUT scale gets large loop corrections and the RG improved effective potential is no longer UFB. However, it generates a large VEV (compared to the EW scale) CCB minimum. If the potential energy of the $\mathrm{CCB}$ minimum is lower than that of the EW minimum, the $\mathrm{EW}$ minimum can tunnel to the CCB minimum. In most of parameter space the tunneling rate is too small and the EW vacuum can live longer than the age of the universe 16, 17]. More precisely, the longetivity of the metastable $\mathrm{EW}$ vacuum puts a constraint $m_{\tilde{t}}\left(M_{Z}\right) \gtrsim \frac{1}{10} M_{3}\left(M_{Z}\right)$ [16], and again the region of parameter space we consider is entirely safe from this bound (nevertheless it tells us that stop masses squared cannot be arbitrarily large and negative at the GUT scale).

A possible problem is that after inflation the universe is likely to settle down in a large VEV CCB vacuum rather than the EW vacuum. This is worrisome since the tunneling rate to the EW vacuum would be very small. However, if the reheating temperature is high enough, the large VEV CCB minimum might disappear in finite temperature effective potential. For a given set of SSBs, there is a minimum reheating temperature above which the large VEV CCB vacuum disappears 18. It depends on how inflation ends and SSBs will constrain compatible inflation scenarios.

In this letter we focused on the SUSY parameters relevant for radiative EWSB and discussion of fine tuning. An interesting signature of this scenario is stop splitting, $m_{\tilde{t}_{1}, \tilde{t}_{2}} \simeq m_{\tilde{t}}\left(M_{Z}\right) \mp m_{t}$, and stops considerably lighter than gluino: $m_{\tilde{t}}\left(M_{Z}\right) \lesssim 0.5 m_{\tilde{g}}$ with $m_{\tilde{g}} \gtrsim 600 \mathrm{GeV}$ (the lighter stop thus can be as light as $130 \mathrm{GeV}$ ). Besides these the scenario has a light Higgsino, a possible candidate for LSP.

Other scalar soft masses squared are unconstrained by considerations of fine tuning and can be positive or even all negative at the GUT scale in complete models. In some SUSY breaking scenarios the sign of the scalar masses squared is not determined [19] while in others it can be fixed and negative. For example, negative scalar masses squared arise in gauge mediation with gauge fields as messengers 20] or one can utilize the minus sign arising in the see-saw mechanism for scalar masses [21]. It is desirable to build fully realistic models of this type in which constrains from CCB can be addressed. In specific scenarios additional potential problems may occur, like negative slepton masses squared at the EW scale since the right-handed sleptons receive contribution only from $M_{1}$. Even if this contribution is large enough to drive sleptons positive, we still can end up with stau LSP.

Nevertheless all the positive features of negative stop masses squared suggest it is worthwhile to seriously search for models which can lead to boundary conditions discussed above.

We thank K. Agashe, A. Falkowski, G. Giudice and R. Rattazzi for discussions. HK thanks CERN and IAS for the hospitality during the visit. This work was supported by the U.S. Department of Energy, grant DEFG02-90ER40542, by the ABRL Grant No. R14-2003012-01001-0, the BK21 of MOE, Korea and the SRC of the KOSEF through the CQUeST of Sogang University with grant number R11-2005-021.

[1] The limit $m_{h} \gtrsim 114.4$ is the limit on the mass of the standard model Higgs boson. However, in most region of parameter space it also applies to the lightest Higgs in MSSM. For more details, see LEP Working Group for Higgs boson searches, LHWG-Note-2005-01.

[2] For a detailed discussion of fine tuning in MSSM and extensive list of references see, D. J. H. Chung, L. L. Everett, G. L. Kane, S. F. King, J. D. Lykken and L. T. Wang, Phys. Rept. 407, 1 (2005).

[3] S. Heinemeyer, W. Hollik and G. Weiglein, Comput. Phys. Commun. 124, 76 (2000).

[4] S. Heinemeyer, W. Hollik and G. Weiglein, Eur. Phys. J. C 9, 343 (1999).

[5] M. Carena et al., Nucl. Phys. B 580, 29 (2000), and references therein.

[6] C. Le Mouel, Phys. Rev. D 64, 075009 (2001).

[7] L. E. Ibanez and C. Lopez, Nucl. Phys. B 233, 511 (1984).

[8] M. Carena, P. Chankowski, M. Olechowski, S. Pokorski and C. E. M. Wagner, Nucl. Phys. B 491, 103 (1997).

[9] K. Choi, K. S. Jeong, T. Kobayashi and K. i. Okumura, arXiv:hep-ph/0508029

[10] R. Kitano and Y. Nomura, Phys. Lett. B 631, 58 (2005).

[11] O. Lebedev, H. P. Nilles and M. Ratz, arXiv:hep-ph/0511320

[12] J. F. Gunion, H. E. Haber and M. Sher, Nucl. Phys. B 306, 1 (1988).

[13] J. A. Casas, A. Lleyda and C. Munoz, Nucl. Phys. B 471, 3 (1996).

[14] J. M. Frere, D. R. T. Jones and S. Raby, Nucl. Phys. B 222, 11 (1983).

[15] J. P. Derendinger and C. A. Savoy, Nucl. Phys. B 237, 307 (1984).

[16] A. Riotto and E. Roulet, Phys. Lett. B 377, 60 (1996).

[17] A. Kusenko, P. Langacker and G. Segre, Phys. Rev. D 54, 5824 (1996).

[18] T. Falk, K. A. Olive, L. Roszkowski, A. Singh and M. Srednicki, Phys. Lett. B 396, 50 (1997).

[19] For recent discussion of negative scalar masses squared in mSUGRA, see J. L. Feng, A. Rajaraman and B. T. Smith, arXiv:hep-ph/0512172

[20] G. F. Giudice and R. Rattazzi, Nucl. Phys. B 511, 25 (1998).

[21] H. D. Kim, Phys. Rev. D 72, 055015 (2005).

[22] Slightly smaller $m_{\tilde{t}}$ is allowed, the actual minimal possible value of $m_{\tilde{t}}$ is not important for our discussion. 
[23] To some extend this is already signaled by bounds on masses of superpartners from direct searches, however the limits on the Higgs mass and the above discussion make this absolutely clear.

[24] To be more precise the generated mixing is somewhat larger than that shown in this equation, since we should minimize the potential at the SUSY scale $\sim m_{\tilde{t}}$ and should not run SSBs all the way to $M_{Z}$ (see Fig. 1). 